\title{
Immunomodulatory Properties of Lactobacillus plantarum NC8 Expressing an Anti-CD11c Single-Chain Fv Fragment
}

\author{
Jing Liu, Guilian Yang, Xing Gao, Zan Zhang, Yang Liu, Xin Yang, Chunwei Shi, Qiong Liu, Yanlong Jiang*, \\ and Chunfeng Wang*
}

College of Animal Science and Technology, Jilin Provincial Engineering Research Center of Animal Probiotics Key Laboratory of Animal Production and Product Quality Safety, Ministry of Education Jilin Agricultural University, Changchun, P.R. China

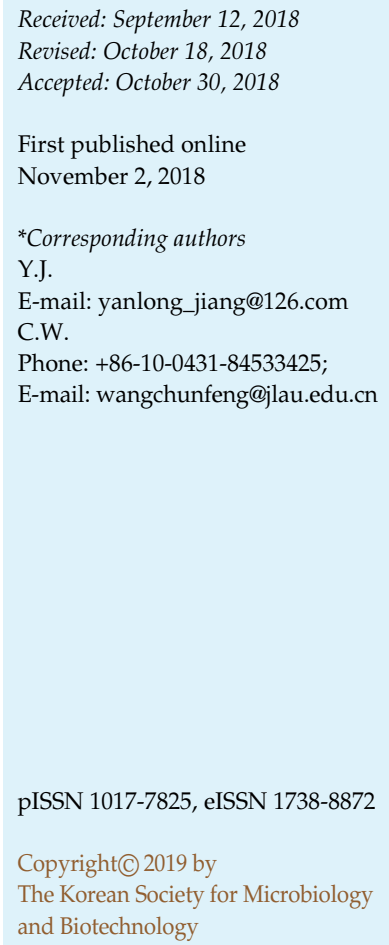

The lactic acid bacteria species Lactobacillus plantarum (L. plantarum) has been used extensively for vaccine delivery. Considering to the critical role of dendritic cells in stimulating host immune response, in this study, we constructed a novel CD11c-targeting L. plantarum strain with surface-displayed variable fragments of anti-CD11c, single-chain antibody (scFv-CD11c). The newly designed L. plantarum strain, named 409-aCD11c, could adhere and invade more efficiently to bone marrow-derived DCs (BMDCs) in vitro due to the specific interaction between scFv-CD11c and CD11c located on the surface of BMDCs. After incubation with BMDCs, the 409-aCD11c strain harboring a eukaryotic vector pValac-GFP could lead to more efficient expression of GFP compared with wild-type strains shown by flow cytometry analysis, indicating the enhanced translocation of pValac-GFP from L. plantarum to BMDCs. Similar results were also observed in an in vivo study, which showed that oral administration resulted in efficient expression of GFP in both Peyer's patches (PP) and mesenteric lymph nodes (MLNs) within 7 days after the last administration. In addition, the CD11c-targeting strain significantly promoted the differentiation and maturation of DCs, the differentiation of $\mathrm{IL}-4^{+}$and IL-17A ${ }^{+}$T helper (Th) cells in MLNs, as well as production of $\mathrm{B}_{2} 20^{+} \mathrm{IgA} \mathrm{g}^{+} \mathrm{B}$ cells in the PP. In conclusion, this study developed a novel DC-targeting L. plantarum strain which could increase the ability to deliver eukaryotic expression plasmid to host cells, indicating a promising approach for vaccine study.

Keywords: Dendritic cell, CD11c, Lactobacillus plantarum, immunomodulatory

\section{Introduction}

DNA vaccination, a type of third-generation vaccine (also called a nucleic acid vaccine or gene vaccine), primarily depends on the injection of a eukaryotic plasmid containing DNA into host cells using an intramuscular strategy. However, this strategy may render the DNA unstable in some cases [1]. Besides, the traditional injection approach makes it inconvenient for application in the field, especially in poultry production, for example. This being the case, the mucosal delivery vectors have been drawing more attention recently due to their orally administrated method of immunization.

Among these vectors, one of the probiotic strains, lactic acid bacteria ( $\mathrm{LAB}$ ), has raised a lot of interest due to its beneficial properties for human health [2]. To date, a large number of protective antigens have been previously delivered by LAB strains, mainly including Lactococcus lactic (L. lactic) [3-5] and Lactobacillus plantarum (L. plantarum) strains [6, 7]. Compared with L. lactic strains, the L. plantarum strains are able to colonize more efficiently in the intestinal tract, which is the first step for efficient antigen production and necessary for subsequent antigen processing in host cells. In addition, the L. plantarum has been studied in great detail for its immunomodulatory effects and adjuvant-like actions $[8,9]$.

Dendritic cells (DCs), belonging to the family of antigenpresenting cells (APCs), play a critical role in both innate 
immunity and adaptive immunity. The recognition function of DCs in innate immunity is based on their ability to recognize pathogenic microorganisms by special receptors $[10,11]$, such as receptors for C-type lectins (DC-SIGN, DEC-205), toll-like receptors (TLR), receptors for the FC portion of antibodies $(\mathrm{FcR})$, complement receptors (CR3 and CR4) [12-15], as well as CD11c, which belong to the heterodimeric receptor family of $\beta 2$-integrins [13]. The immature DCs capture and internalize specific antigens, followed by expression of co-stimulatory molecules and maturation, and then present the processed antigens to stimulate $\mathrm{T}$ cell differentiation and $\mathrm{B}$ cell production, which play important roles in host immune response.

To enhance the ability of DCs to capture and process foreign antigens, one of the strategies is to make use of the surface markers of DCs. For example, a surface displayed single-chain antibody against DEC-205 (scFv-DEC205) on L. plantarum significantly enhanced the internalization of bacterium and plasmid transfer to DCs both in vitro and in vivo [16]. In this study, we constructed a novel L. plantarum strain with surface displayed, single-chain antibody against $\mathrm{CD} 11 \mathrm{c}$ (scFv-CD11c). This newly designed strain efficiently attaches and invades DCs due to the specific interaction between scFv-CD11c and surface CD11c on DCs, followed by increased plasmid transfer to cells both in vitro and in vivo. Furthermore, the DC-targeting L. plantarum strains could efficiently stimulate the maturation of DCs in vivo, as well as the differentiation of $\mathrm{T}$ cells and production of $\mathrm{B} 220^{+} \mathrm{B}$ cells, indicating its potential for use in further vaccine study.

\section{Materials and Methods}

Construction of the Recombinant Strain Expressing scFv-CD11c

The strains and plasmids used in this study are listed in Table 1. L. plantarum strain NC8 (CCUG 61730) and the Escherichia coliLactobacillus shuttle vector pSIP409 [17] were kindly provided by
Professor A. Kolandaswamy (Madurai Kamaraj University, India). The pValac-GFP plasmid was kindly provided by Anderson Miyoshi [18]. LB medium was used for the culture of E. coli strains, whereas MRS medium was used for L. plantarum. Whenever necessary, erythromycin $(50 \mathrm{ng} / \mathrm{ml})$ and chloramphenicol $(10 \mu \mathrm{g} / \mathrm{ml})$ were used for antibiotics selection.

The scFv-CD11c sequences (cloneN418, Gene ID: 16411) were optimized for expression in L. plantarum and synthesized by Genewiz Co. (China). LysM anchoring sequences (Sequence: WP_015380879.1, source: 30-74) [16] were used to anchor the scFvCD11c fragment on the surface of L. plantarum. In detail, the LysM-scFv-CD11c fragments were synthesized and digested with NcoI and HindIII, then the pSIP409 was also digested by the same enzymes. After ligation, the acquired plasmid named pSIP409aCD11c was electroporated into L. plantarum NC8 strain, yielding NC8 (pSIP409-aCD11c), according with the previously described methods [19]. In brief, $1 \mu \mathrm{g}$ plasmid pSIP409-aCD11c was added to $100 \mu \mathrm{lNC} 8$ competent cells in cuvettes with a 0.2 -cm electrode gap (Flowgen, UK) and was electroporated using a Gene Pulser electroporator (Bio-Rad, Hemel, UK). The electroporation parameters were $2.0 \mathrm{kV}, 400 \Omega$ parallel resistance, and $25 \mu \mathrm{F}$ capacitance after $10 \mathrm{~min}$ on ice. Next, the cells were added to $600 \mu \mathrm{l}$ MRS broth and incubated $3 \mathrm{~h}$ at $37^{\circ} \mathrm{C}$. Finally, dilutions of bacteria were plated onto MRS agar containing erythromycin and selected positive clones. To further determine the ability of our strain to deliver eukaryotic expression plasmid, pValac-GFP which could express GFP protein under CMV promoter was transformed into NC8 (pSIP409-aCD11c), yielding a double antibiotic selected strain NC8 (pSIP409-aCD11c, pValac-GFP).

\section{The Expression of aCD11c}

The recombinant NC8 (pSIP409-aCD11c) strains were cultured using MRS medium to examine the expression of aCD11c, a NC8 (pSIP409) strain was also included as negative control. To induce aCD11c expression, SppIP, was added into the medium at a final concentration of $50 \mathrm{ng} / \mathrm{ml}$ when the cultures reached a $600 \mathrm{~nm}$ absorbance (A600) of 0.3 . After induction at $30^{\circ} \mathrm{C}$ for $10 \mathrm{~h}$, the cultures were adjusted to the same $\mathrm{A} 600$ values, harvested by centrifugation at $5,000 \times g$ for $10 \mathrm{~min}$ at $4^{\circ} \mathrm{C}$, and then washed twice times using $500 \mu \mathrm{l}$ of cold, phosphate-buffered saline (PBS)

Table 1. Bacterial strains and plasmids used in this study.

\begin{tabular}{llc}
\hline \multicolumn{1}{c}{ Plasmids or strains } & \multicolumn{1}{c}{ Description } & Source \\
\hline pSIP409 & p256rep/pUC(pGEM),PsppIP,sppR,K, Em ${ }^{\mathrm{r}}$ & {$[17]$} \\
pSIP409-aCD11c & PsppIP,sppR,K,aCD11c, Em ${ }^{\mathrm{r}}$ & This study \\
E. coli TOP10 & Host strain & TaKaRa \\
L. plantarum NC8(CCUG 61730) & Host strain, Plasmid-free, silage isolate & {$[17]$} \\
NC8(pSIP409) & L. plantarum NC8 with pSIP409 & This study \\
NC8(pSIP409-aCD11c) & L. plantarum NC8 with pSIP409-aCD11c & This study \\
409/pValac-GFP & L. plantarum NC8 with pSIP409 and pValac-GFP & This study \\
409a/pValac-GFP & L. plantarum NC8 with pSIP409-aCD11c and pValac-GFP & This study \\
\hline
\end{tabular}


and resuspended in $1 \mathrm{ml}$ of PBS containing $1 \mathrm{mM} \mathrm{CaCl}_{2}, 1.1 \mathrm{M}$ sucrose, $0.5 \mathrm{M} \mathrm{MgCl}_{2}, 100 \mu \mathrm{g}$ of RNase (Sigma Chemicals, USA), $50 \mu \mathrm{g}$ of DNase (Sigma), and a cocktail of anti-proteases (Complete; Roche Molecular), and then $5 \mathrm{mg}$ of lysozyme (Sigma) plus $100 \mu \mathrm{g}$ of mutanolysin (Sigma) were also added. The digestion reactions were performed for $30 \mathrm{~min}$ at $37^{\circ} \mathrm{C}$ with shaking at 120 rpm and then centrifugation for $30 \mathrm{~min}$ at $10,000 \times g$. The supernatants were acquired [20] and the protein samples from cell wall fractions were then subjected to western blotting assay using His-tag antibody (CWBIO, China) as primary antibody and HRPconjugated secondary antibody (Abbkine, USA). Visualization of the immunobinding was conducted by enhanced chemiluminescence (ECL) using an ECL Plus detection kit (Thermo Scientific).

\section{Detection of scFv-CD11c at the Surface of L.plantarum}

The strains were cultured and induced by SppIP according to the above methods. The bacterial cells, $1 \times 10^{5}$ colony forming units (CFU) of each strain, were harvested and washed three times using cold PBS, and then incubated using Anti His-tag Mouse Monoclonal antibody (CWBIO, China) as primary antibody and Fluorescein (FITC)-conjugated Affinipure Goat Anti-Mouse IgG $(\mathrm{H}+\mathrm{L})$ (Proteintech, USA) as secondary antibody, respectively, as previously described [21]. The FlowJo 7.6.1 software was used to analyze the data.

\section{Adhesion and Invasion Characteristics of NC8 (pSIP409-aCD11c) to BMDCs In Vitro}

Five-to-six-week-old C57BL/6 mice were purchased from Beijing HFK Bioscience Co., Ltd., China. Bone marrow-derived DCs (BMDCs) were acquired from mice bone marrow precursors cultured with GM-CSF and IL-4 (PeproTech, USA) according to the method described previously [22]. At day 8 , the cells were collected and distributed in a P24 dish $\left(2 \times 10^{5}\right.$ cells per well $)$ and cultured for further $24 \mathrm{~h}$. The induced strains were then added to the cells with a multiplicity of infection (MOI) of $10^{3}$ bacteria per cell in RPMI 1640 medium (Sigma-Aldrich, USA) without antibiotics.

To determine the bacteria adhesion ability, the cells were washed carefully with PBS for six times after $2 \mathrm{~h}$ of incubation, followed by disruption with sterile PBS containing $0.2 \%$ Triton X-100 for $10 \mathrm{~min}$. The CFU were then counted after plating serial dilutions on erythromycin selection plates.

To determine the bacteria invasion, the cells after co-incubation with recombinant strains as described above were washed completely and treated with gentamicin $(500 \mu \mathrm{g} / \mathrm{ml})$ for an additional $2 \mathrm{~h}$ to kill any extracellular bacteria. After further PBS washing three times, the cell disruption and counting were performed as mentioned above. A competitive assay was also included to evaluate the specific interaction between recombinant scFv-CD11c and CD11c on DCs. In detail, the monoclonal antimouse CD11c antibody (Bioss, China) was incubated with BMDCs at a final concentration of $2 \mu \mathrm{g} / \mathrm{ml}$ for $1 \mathrm{~h}$ before incubation with bacteria. Then the cell invasion assay was performed as described above. All experiments were conducted in triplicate. The adhesion and invasion ratios were equal to the CFU of disrupting cells using Triton X-100/CFU of the original inoculated bacterial culture.

To further observe the cellular invasion of recombinant strains, the BMDCs incubated with bacteria as mentioned above were fixed with $2.5 \%$ Gluta at room temperature for $1 \mathrm{~h}$, followed by transmission electron microscopy (TEM) assay (Harbin Veterinary Research Institute, Chinese Academy of Agricultural Sciences, China).

\section{Plasmid Delivery to BMDCs}

To determine the plasmid delivery ability of our recombinant L. plantarum strains, the NC8 (pSIP409-aCD11c, pValac-GFP) strains were incubated with BMDCs with a MOI of 100 in RPMI 1640 medium (Sigma-Aldrich, USA) without antibiotics and incubated for $36 \mathrm{~h}$. Then a FACS assay was performed to evaluate the plasmid transfer. In detail, $10 \mu \mathrm{l}$ of APC hamster anti-mouse CD11c antibody was added to cell suspensions of treated BMDCs and incubated for $40 \mathrm{~min}$ at $4{ }^{\circ} \mathrm{C}$ in dark. After three washings with FACS buffer (PBS, 1\% FCS, 0.09\% sodium azide) (Sigma, USA), the cells were sorted by APC-CD11c gate and GFP expression using BD FACS LSRFortessa flow cytometer) to determine the percentages of GFP expression in the CD11 $\mathrm{c}^{+}$cells. FlowJo 7.6.1 software was used to analyze the data.

Plasmid Transfer In Vivo by Recombinant L. plantarum Strains Animal studies were approved by the Jilin Agriculture University Animal Committee (JLAU04201801). Twenty-seven 5to-6-week-old BALB/c female mice were purchased from Beijing HFK Bioscience Co., Ltd., China, and divided into three groups, with nine mice in each group. The mice were orally administrated with either NC8 (pSIP409, pValac-GFP) or NC8 (pSIP409-aCD11c, pValac-GFP) strains at the dose of $10^{9} \mathrm{CFU}$ bacteria/100 $\mu \mathrm{l} /$ mouse over 3 consecutive days (days 1,2, and 3) [16], and mice treated with saline were also included as a negative control. At days 1, 3, and 7 after the last administration, three mice from each group were sacrificed and the single cell suspensions were prepared from Peyer's patches (PPs) and mesenteric lymph node (MLN) and subjected to FACS assay to detect the expression of GFP proteins.

\section{Immunological Modulation Effects by Recombinant L. plantarum} Strains

An additional fifteen BALB/c mice were randomly divided into three groups and orally administrated with the same strains mentioned above. The mice were administrated with each strain at days 1, 2, 3, 15, 16, and 17. One week later, all mice were sacrificed and subjected to FACS assay to determine the maturation of DCs, and differentiation of $\mathrm{T}$ cells and B cells.

\section{Flow Cytometer Assay}

The single cell suspensions were prepared from PPs and MLNs using a sterile stencil and suspended in RPMI 1640 with 1\% penicillin and streptomycin. Subsequently, cell suspensions were 
filtered via a 70- $\mu \mathrm{m}$ nylon cell strainer (BD Biosciences, USA), centrifuged at $500 \times g$ for $5 \mathrm{~min}$ at $4^{\circ} \mathrm{C}$, washed with cold PBS, and re-suspended in complete RPMI 1640 (containing 10\% FBS, penicillin and streptomycin). Then the following antibodies were used in a FACS assay of DC phenotypes from MLN, including APC labeled anti-mouse CD11c, PerCP-Cy5.5 labeled anti-mouse CD80, PE labeled anti-mouse CD83 and their respective isotype control antibodies. For $\mathrm{T}$ cell intracellular cytokines, portions of the MLN suspensions were cultured in 48-well polystyrene microtiter plates, and the cells were diluted to $2 \times 10^{6}$ cells $/ \mathrm{ml}$ in complete RPMI 1640. A total of $4 \mu$ l Leukocyte Activation Cocktail with BD GolgiPlug (BD Pharmingen) $(1 \mu \mathrm{M})$ was added to each well, which contained $2 \times 10^{6}$ cells, on a sterile super clean bench, and the cells were cultured at $37^{\circ} \mathrm{C}, 5 \% \mathrm{CO}_{2}$ for 6 hours. After 6 hours, the cells were washed and stained with PerCP-Cy5.5conjugated antibodies for CD3e and FITC-conjugated antibodies for CD4 (BD Pharmingen). For B cells, PP cells were labeled using APC labeled anti-mouse B220 directly. The cells were then washed, fixed and permeabilized with the BD Cytofix/Cytoperm Plus/Fixation/Permeabilization Kit (BD Pharmingen) according to the manufacturer's instructions. All the cells of MLN were then stained with PE-conjugated anti-IFN- $\gamma$, APC-conjugated anti-IL-4 and PE-cy7-conjugated anti-IL-17A, and PP cells with FITC labeled anti-mouse IgA as well as their respective isotype control antibodies (BD Pharmingen). The cells were analyzed by FACS [23].

\section{Statistical Analysis}

The significance of the data was assessed through GraphPad Prism 5 software. The data were presented as the mean \pm standard error of the mean (S.E.M.) and were analyzed by one-way ANOVA (Dunnett's multiple comparison test) in at least three independent experiments.

\section{Results}

\section{Construction of L. plantarum Strains with Surface Displayed scFv-CD11c}

A scFv-CD11c fragment containing LysM anchoring sequences and His-tag (Fig. 1A) was inserted into vector pSIP409, yielding pSIP409-aCD11c. To determine the surface localization of the aCD11c, the cell wall fractions were extracted and subjected to western-blotting analysis by His-tag antibody. A 38-kDa band of scFv-CD11c (Fig. 1B, lane 2) was observed in NC8 (pSIP409aCD11c), whereas no band was observed in the NC8 (pSIP409) negative control. In addition, the presence of $\mathrm{scFv}-\mathrm{CD} 11 \mathrm{c}$ protein on the bacterial surface was also confirmed by FACS analysis (Fig. 1C).

\section{Adhesion and Invasion Assay of L. plantarum Expressing scFv-CD11c in BMDCs}

Attachment followed by invasion processes are critical for DCs to capture and acquire specific antigens, which is also the first step of adaptive immune response. We determined the adhesion and invasion ability of wild-type L. plantarum NC8 strain and the newly constructed strain with surface displayed scFv-CD11c. The results showed that the wild-type NC8 could attach to BMDCs at a ratio of about $23 \%$, whereas the expressed $\mathrm{scFv}-\mathrm{CD} 11 \mathrm{c}$ significantly increased this ratio to about $35 \%$ (Fig. 2A). To note, the increased attachment was dramatically decreased during the competitive assay in which the commercial anti-CD11c antibody was present before co-incubation, indicating that the increased cellular adhesion was caused by the presence of $\mathrm{scFv}-\mathrm{CD} 11 \mathrm{c}$ on the bacterial surface. Similar results were also observed in the cellular invasion study (Fig. 2B), and the presence of scFv-CD11c significantly increased the numbers of bacteria in BMDCs, whereas the
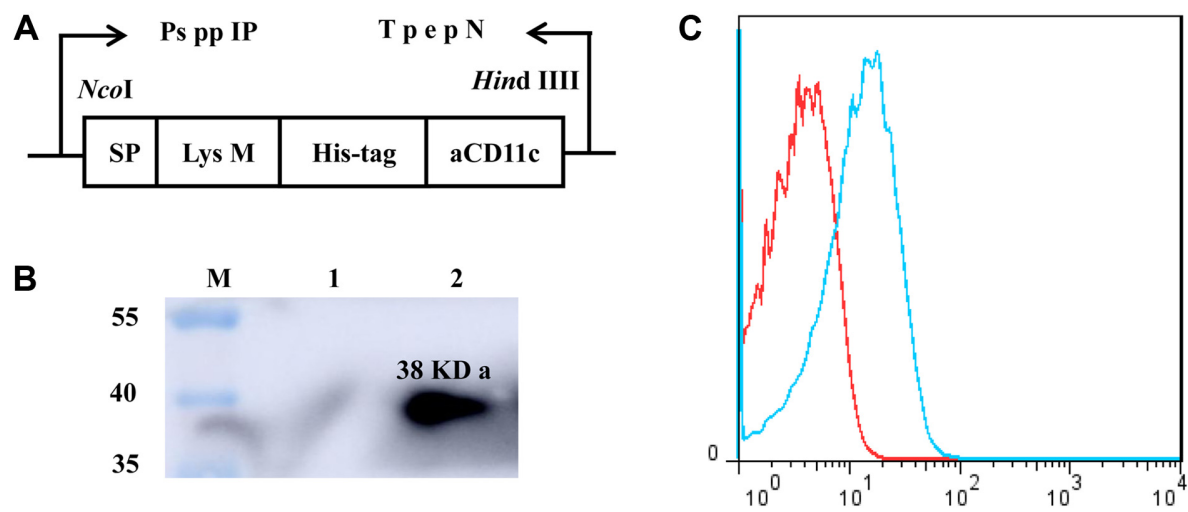

Fig. 1. Characterization of L. plantarum expressing scFv-CD11c.

(A) Expression cassettes containing the aCD11c fragment encoding anti-mouse CD11c ScFv under the control of the inducible $\mathrm{P}_{\text {sppIP }}$ promoter. An $\mathrm{N}$-terminal signal sequence (SP) was contained for secreting protein, and a LysM domain was contained for displaying on the surface of L. plantarum and a His-tag was used for western blot detection. (B) The expression of scFv-CD11c in NC8 (pSIP409-aCD11c) strains was determined via western blotting. M: prestained marker, lane 1: NC8 (pSIP409), lane 2: NC8 (pSIP409-aCD11c) (38 kDa). (C) Flow cytometry analysis of the expression of scFv-CD11c at the surface of L. plantarum (the red represents NC8 (pSIP409) strains, the blue represents the NC8 (pSIP409-aCD11c) strains). 
A
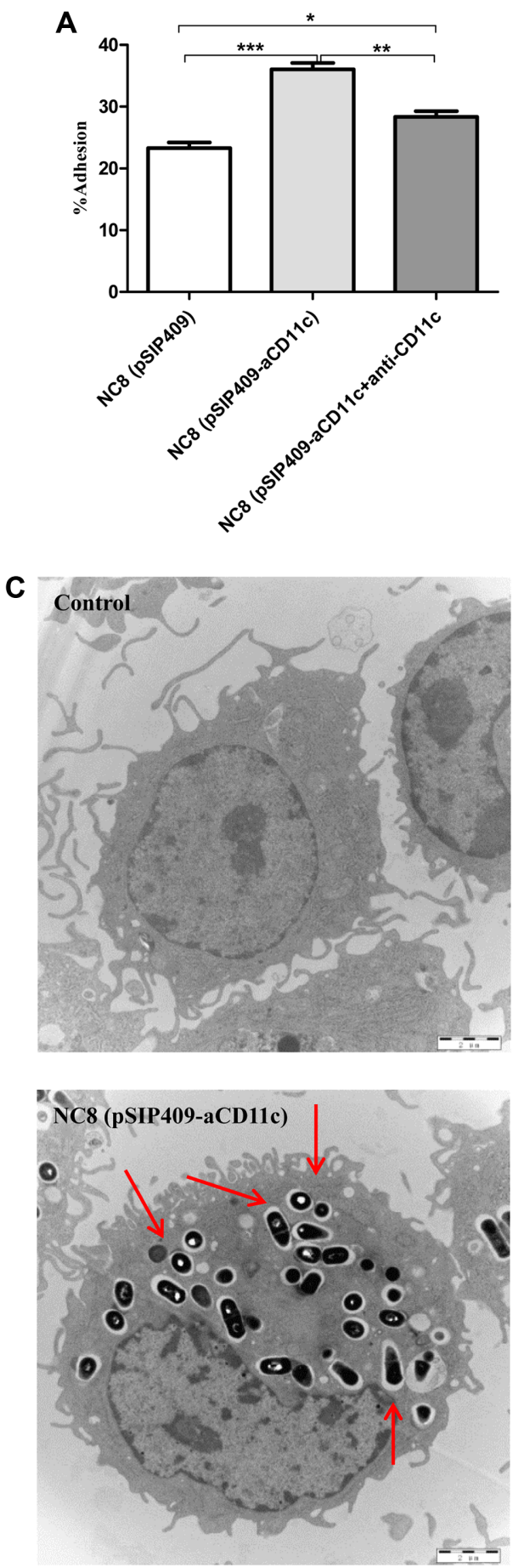
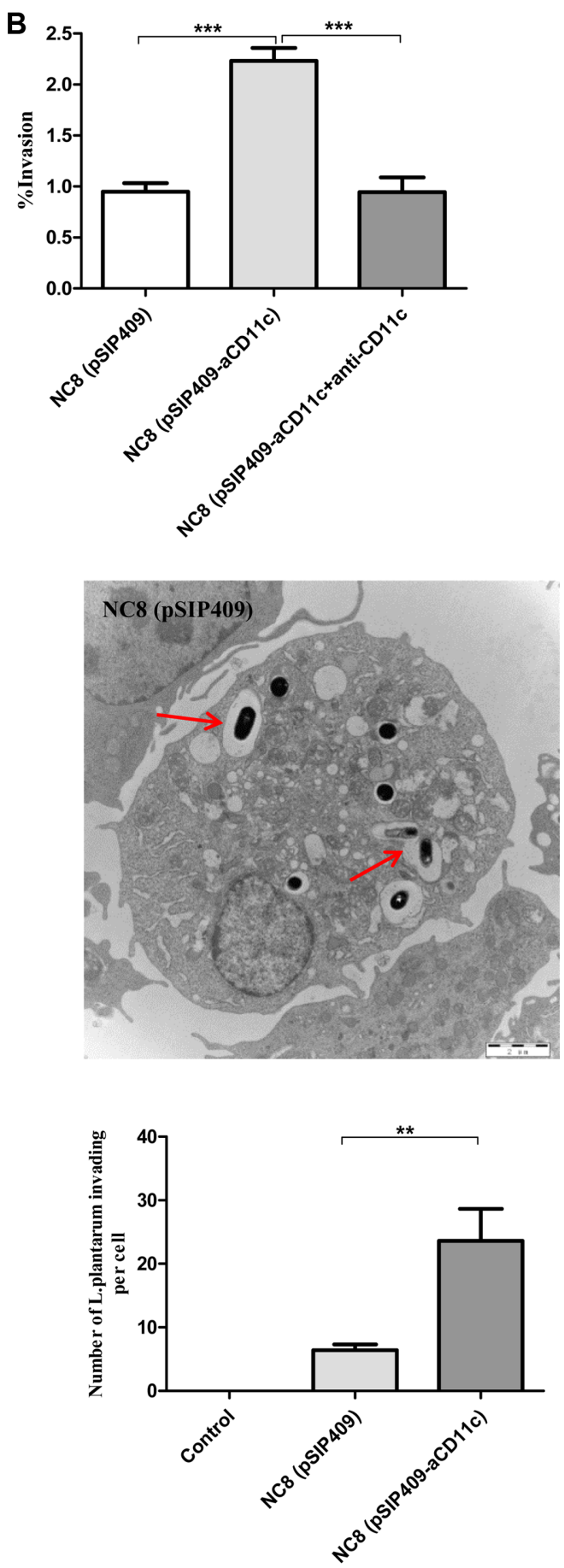

Fig. 2. Adhesion and invasion assays of the strains to BMDCs were observed by transmission electron microscopy (TEM).

(A) The adhesion assay was consistent with materials and methods mentioned above. An adhesion test was acquired by disrupting the cells using Triton X-100. Adhesion ratio $=$ CFU of disrupting cells using Triton X-100/CFU of original inoculated bacterial culture. (B) The invasion rates of BMDCs were detected by a plating assay. Invasion ratio = CFU of disrupting cells using Triton X-100/CFU of original inoculated bacterial culture. (C) TEM analysis. The strains were co-cultured with BMDCs for two hours, and cells were fixed and visualized by TEM. The bacteria in the cytoplasm were indicated by red arrows (magnification at 10,000x, scale bars represent $2.0 \mu \mathrm{m}$ ). Normal BMDCs were included as control. The data are displayed as the mean \pm S.E.M. of triplicate tests $(n=3$ in each group), and the statistical significance was calculated by one-way ANOVA test $\left({ }^{*} p<0.05,{ }^{* *} p<0.01\right.$, and $\left.{ }^{* * *} p<0.001\right)$. 
existence of anti-CD11c antibody alleviated these results to a normal level.

A TEM assay was then conducted to further confirm the increased cellular entrance of our recombinant L. plantarum strains (Fig. 2C). As demonstrated, a couple of NC8 wild type strains were found in BMDCs, whereas a dramatically increased percentage of cellular bacteria were observed in scFv-CD11c expressing strains, indicating the enhanced cellular intake ability due to the presence of scFv-CD11c.

\section{Effect of aCD11c Expression on Plasmid Transfer to BMDCs}

Since we had observed the increased cellular invasion of NC8 (pSIP409-aCD11c) strains, the next question was whether they could increase the plasmid transfer ability to host cells, and this could be an indicator for its future application in DNA vaccine study. A shuttle eukaryotic plasmid pValac-GFP was then transformed into NC8 strains harboring either empty vector pSIP409 or pSIP409-aCD11c. After incubation with BMDCs as mentioned
A

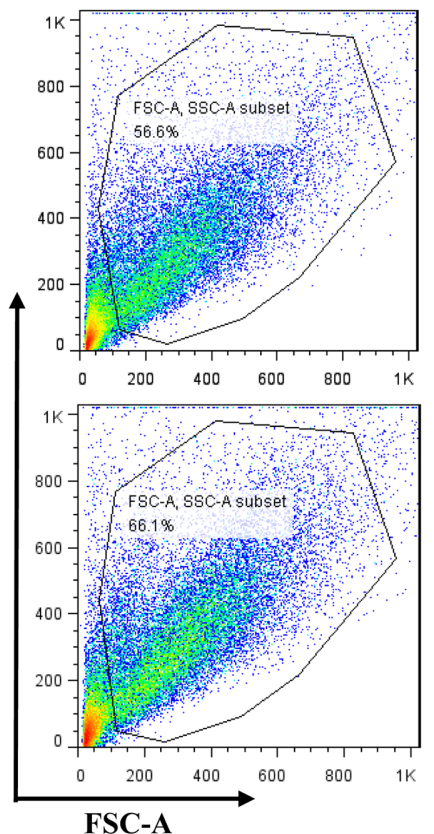

B

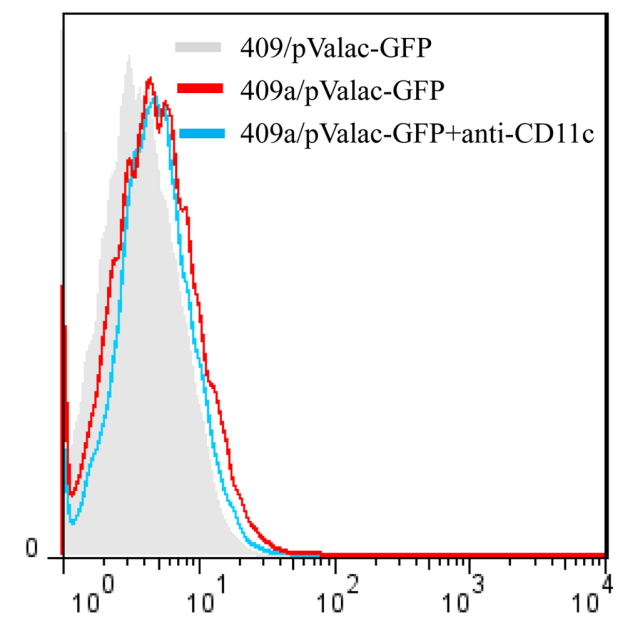

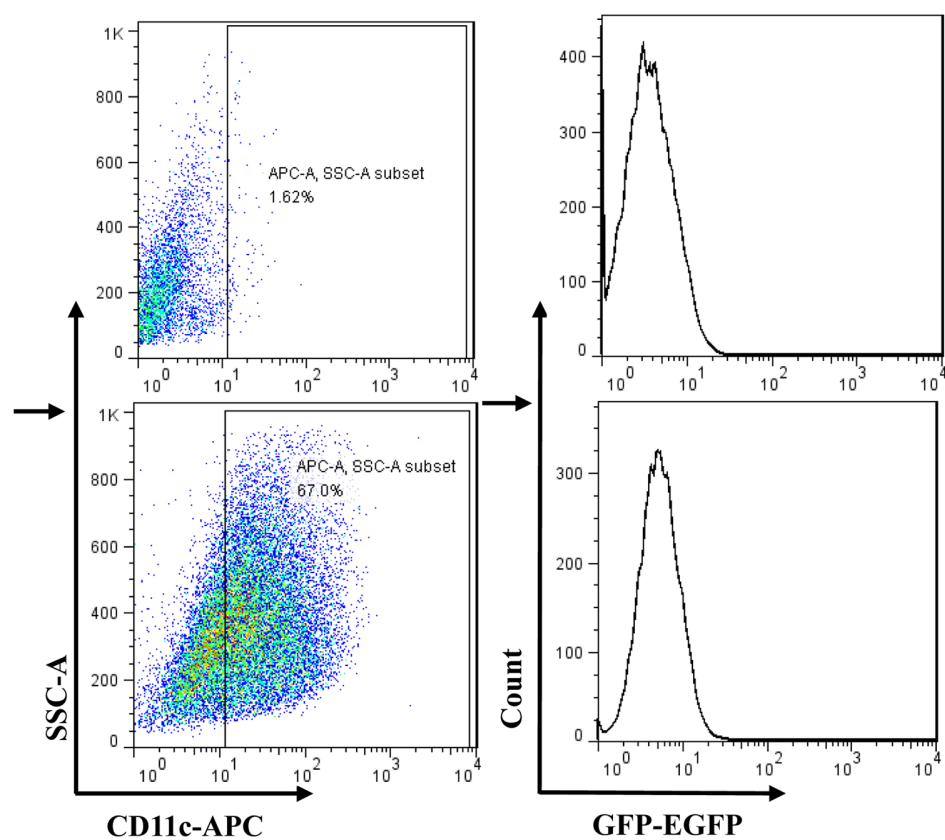

GFP-EGFP

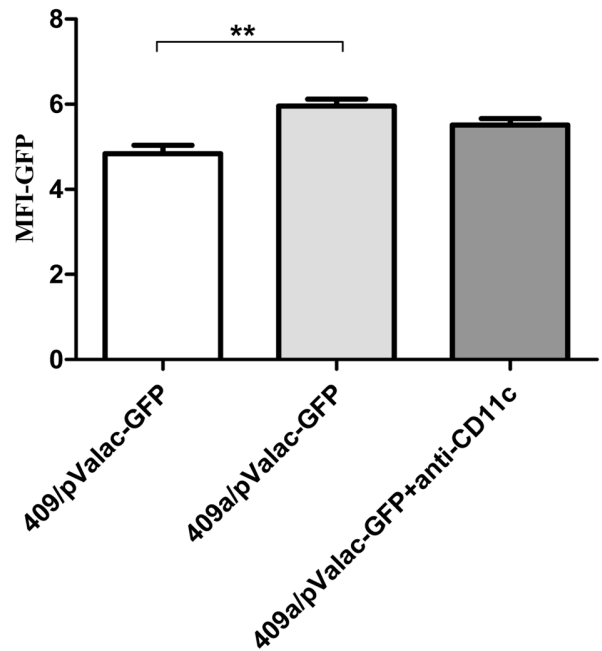

Fig. 3. Expression of $C D 11 c^{+} G F P^{+}$in BMDCs after co-culturing with 409/pValac-GFP and 409a/pValac-GFP strains for $36 \mathrm{~h}$.

(A) The $\mathrm{GFP}^{+}$gating strategy of CD11c ${ }^{+}$DCs. Isotype control was also included. (B) Median fluorescent intensities (MFIs) of GFP in BMDCs were evaluated via flow cytometry. The data are presented as the mean \pm S.E.M. of triplicate tests, and the statistical significance of the differences between groups was analyzed via one-way ANOVA, assuming a Gaussian distribution, followed by Dunnett's test $\left({ }^{* *} p<0.01\right)$. 
above, the BMDCs were analyzed by FACS assay (Fig. 3A) to determine the median fluorescent intensities (MFIs) of GFP in $\mathrm{CD} 11 \mathrm{c}^{+}$cells. And the results demonstrated that the presence of
scFv-CD11c significantly increased the MFI of GFP in BMDCs (Fig. 3B), indicating the increased plasmid transfer ability due to scFv-CD11c.

A
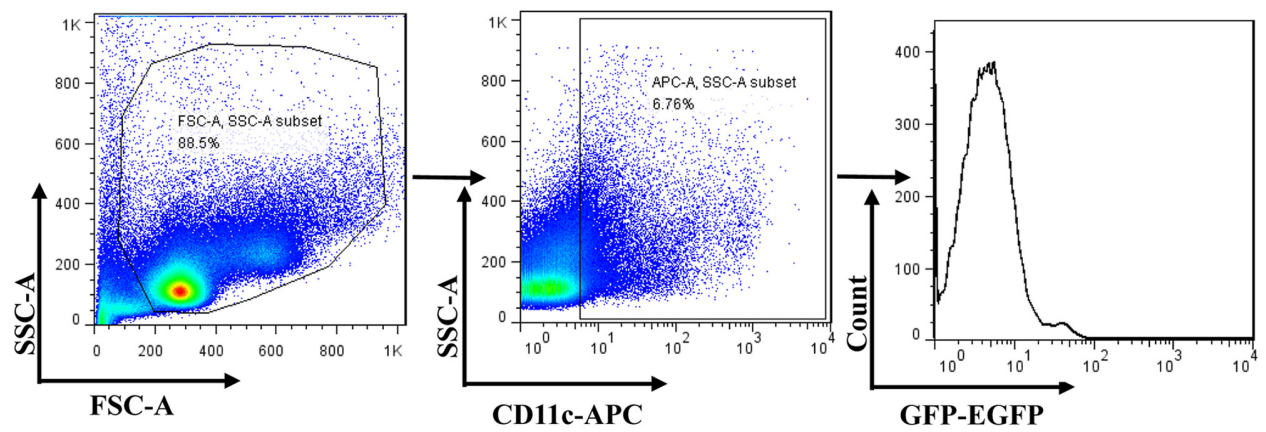

B
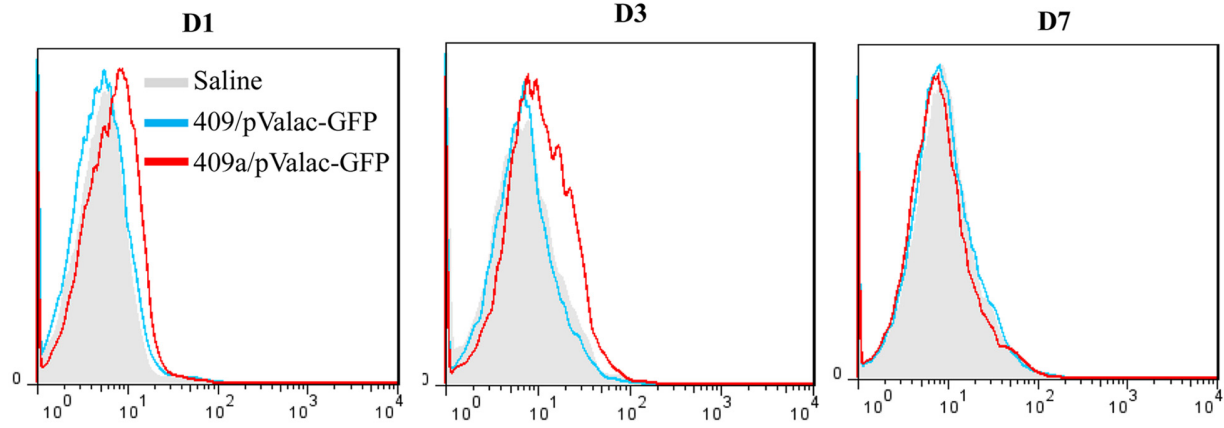

PP
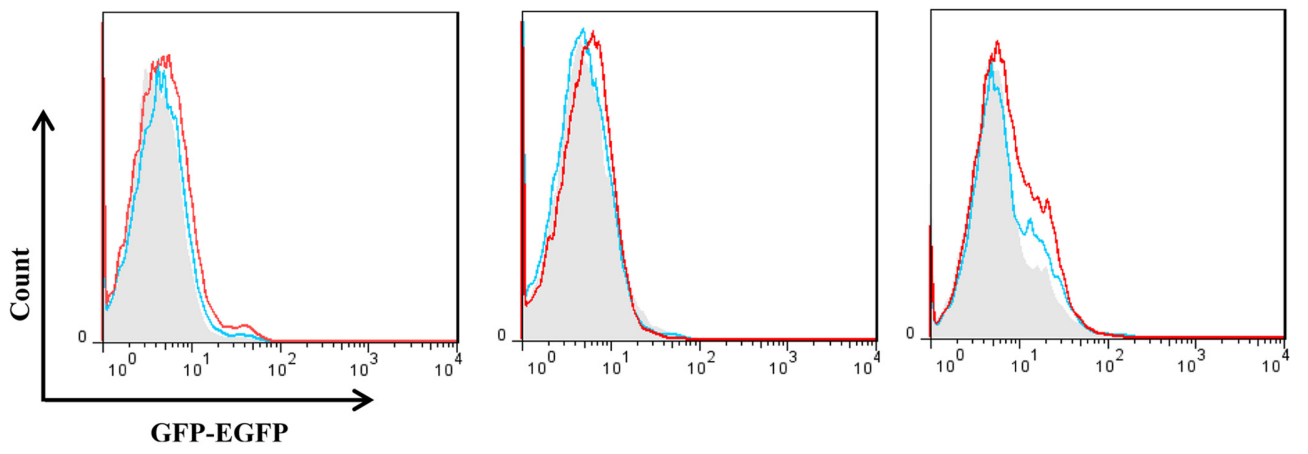

MLN
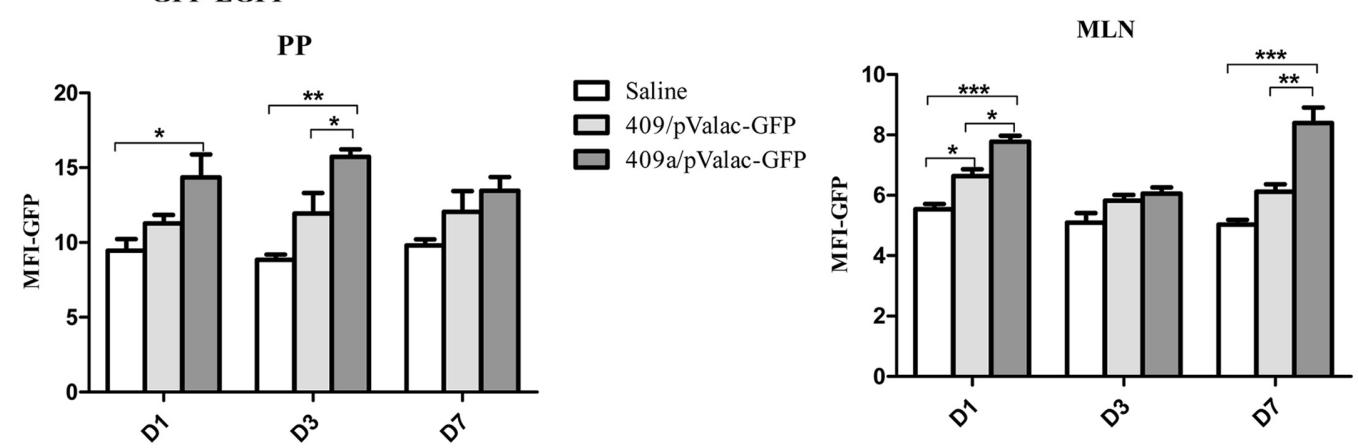

Fig. 4. Effect of GFP expression of L. plantarum expressing scFv-CD11c on CD11c ${ }^{+}$DCs at day 1, day 3 and day 7 (D1, D3, and D7) after the last administration in PP and MLN cells.

(A) The $\mathrm{GFP}^{+}$gating strategy of CD11c ${ }^{+}$DCs in vivo. (B) Median fluorescent intensities (MFIs) of GFP of 409/pValac-GFP and 409a/pValac-GFP strains in PP and MLN cells were analyzed through flow cytometry. The statistical significance was calculated by one-way ANOVA test $\left({ }^{*} p<0.05\right.$, ${ }^{* *} p<0.01$, and $\left.{ }^{* * *} p<0.001\right)$. 


\section{Increased Plasmid Transfer Ability In Vivo Caused by Presence of scFv-CD11c}

To further determine whether the presence of scFv-CD11c could increase the plasmid transfer ability in vivo, both NC8 (pSIP409) and NC8 (pSIP409-aCD11c) strains harboring plasmid pValac-GFP were orally administrated to mice, and the PP and MLN samples were collected at day 1,3, and 7 after immunization and subjected to FACS by gating the $\mathrm{CD} 11 \mathrm{c}^{+} \mathrm{DCs}$ and GFP expression (Fig. 4A). Interestingly, the MFI of GFP of CD11 ${ }^{+}$DCs in PPs were significantly increased at day 3 compared with wildtype strains (Fig. 4B), whereas the MFI of GFP of CD11 ${ }^{+}$DCs in MLN were significantly increased at day 1 and day 7, particularly at day 7 (Fig. 4B). One of the possible explanations was that the DCs in PPs captured the $\mathrm{GFP}^{+}$L. plantarum at day 3 and then migrated to MLN at day 7.

\section{DC-Targeting L. plantarum Stimulated the Differentiation} and Maturation of DCs and the Activation of T Cells

In the mouse model, the expression of the surface markers CD11c, CD80 and CD83 of DCs in MLN were determined by
FACS (Fig. 5A). In general, the presence of scFv-CD11c resulted in increased percentages of $\mathrm{CD} 11 \mathrm{c}^{+} \mathrm{CD} 80^{+} \mathrm{DCs}(\mathrm{P}=0.0004)$ and

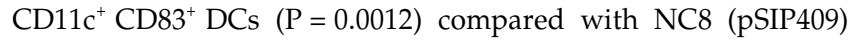
(Fig. 5B), indicating that the NC8 (pSIP409-aCD11c) strains could dramatically stimulate the differentiation and maturation of DCs in vivo, which were important for humoral immune response. Since the next step after the maturation of DCs would be the stimulation of T cells, we then determined the IFN- $\gamma$, IL-4, and IL17A-producing T cells in MLNs (Fig. 6A). The results showed that our newly constructed strains significantly increased the IL- $4^{+}$and IL-17A ${ }^{+}$T cells compared with NC8 (pSIP409) (Fig. 6B), indicating that the NC8 (pSIP409-aCD11c) biased the T cells to Th2 and Th17 subtypes.

\section{Increased Proportions of $\mathrm{B}^{220^{+}} \mathrm{IgA}^{+} \mathrm{B}$ Cells Stimulated by NC8 (pSIP409-aCD11c) Strains}

After the differentiation of $T$ cells, the next step for humoral immune response was the activation of B cells which play a critical role in the production of antibodies. In this case, we determined the percentages of $\mathrm{B}_{2} 20^{+} \mathrm{IgA}^{+} \mathrm{B}$ cells in PPs by FACS

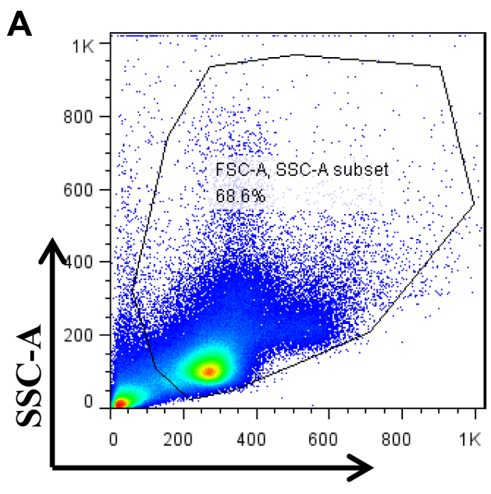

FSC-A

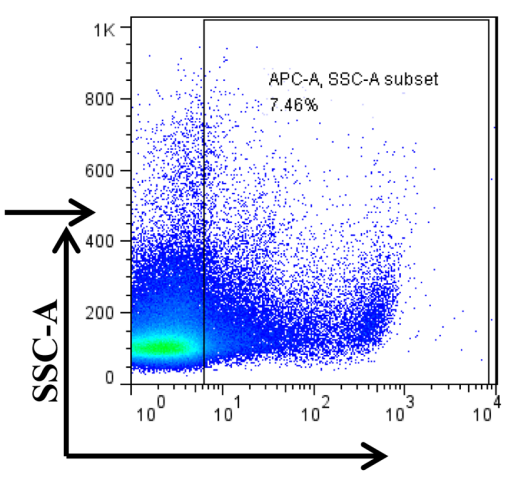

CD11c-APC

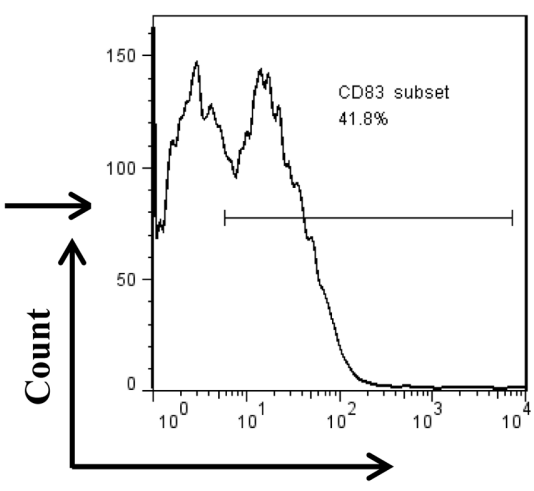

CD83-PE

B
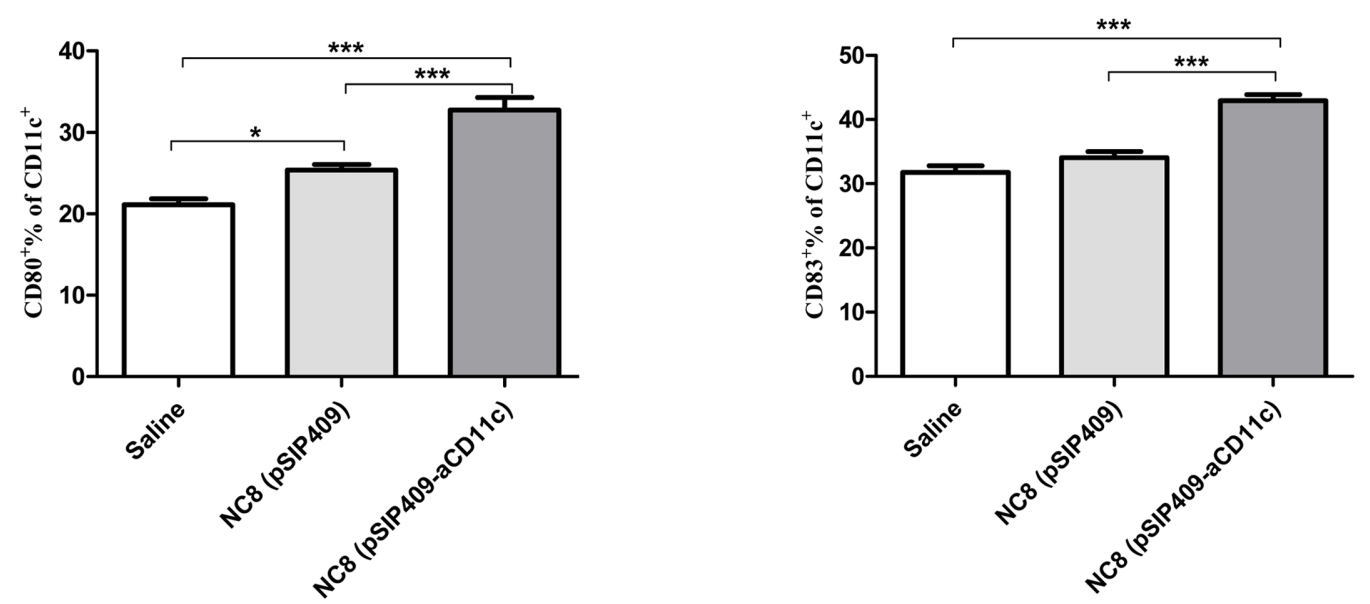

Fig. 5. Expression levels of CD11c, CD80 and CD83 at the surface of DCs.

(A) The DC gating strategy. (B) Production of CD11c, CD80 and CD83 in MLNs were analyzed by flow cytometry. The statistical significance was calculated by one-way ANOVA test ${ }^{*} p<0.05$ and $\left.{ }^{* * *} p<0.001\right)$. 
A

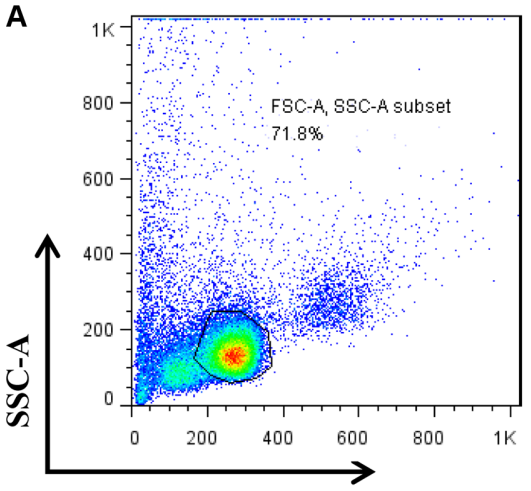

FSC-A
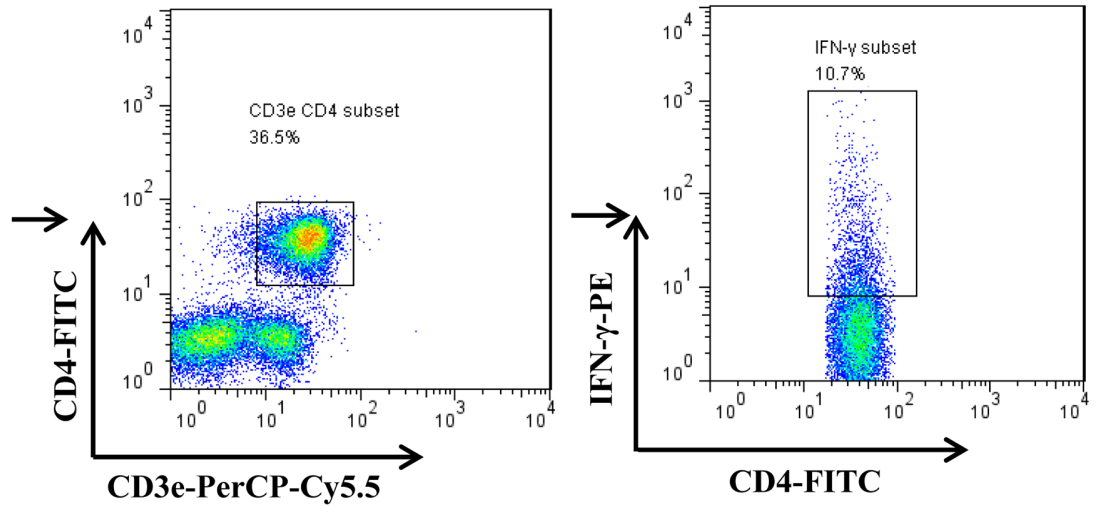

B

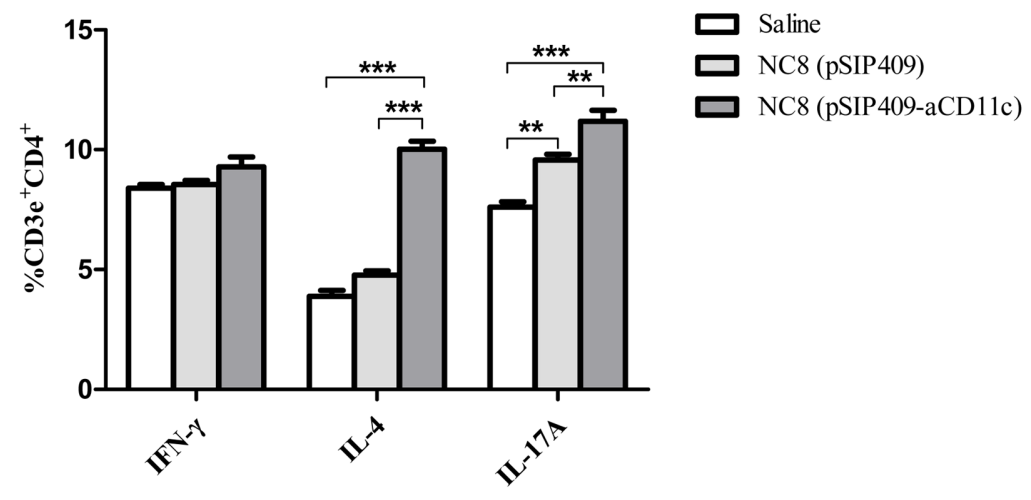

Fig. 6. $\mathrm{CD}^{+} \mathrm{T}$ cell cytokine responses after NC8 (pSIP409-aCD11c) vaccination.

(A) Gating strategy for $\mathrm{CD} 4^{+} \mathrm{T}$ cell intracellular cytokines. (B) The cytokine frequencies of IFN- $\gamma$, IL-4, and IL-17A in CD3e ${ }^{+} \mathrm{CD} 4^{+}$MLN cells were evaluated. Data are expressed as the mean \pm S.E.M. of triplicate tests $(n=5$ mice in each group), and the statistical significance of the differences between groups was assessed by using one-way ANOVA ${ }^{* *} p<0.01$ and $\left.{ }^{* * *} p<0.001\right)$.

(Fig. 7). As demonstrated, the presence of scFv-CD11c significantly stimulated the production of $\mathrm{B}_{2} 20^{+} \mathrm{IgA}^{+} \mathrm{B}$ cells compared with the saline and wild-type strains.

\section{Discussion}

LAB are generally considered as one of the most promising candidates for the development of new and safe vaccines. Both heterologous proteins and plasmid DNA have been delivered by $\mathrm{LAB}$ to host in a number of studies. In particular, the L. lactic strains either expressing invasive protein such as FnBPA [4, 5] or wild-type strains [24, 25] were reported to deliver plasmid to host cells. On the other hand, the L. plantarum strains with surface displayed scFvDEC205 were also observed to deliver DNA to dendritic cells in vitro and in vivo [16]. Considering the fact that L. plantarum could colonize more efficiently at the intestinal positions which will benefit its induced immunological response, we designed a novel L. plantarum strain with DCtargeting properties by expressing $\mathrm{scFv}-\mathrm{CD} 11 \mathrm{c}$ on surface.
In comparison with a previous study [16] in which the scFv-DEC205 was expressed on the surface of L. plantarum strains by three different anchoring sequences, including Lip, CWA and LysM, we made use of the LysM anchoring region to express $\mathrm{scFv}-\mathrm{CD} 11 \mathrm{c}$ since the LysM sequences were found to be the most efficient regarding the expression of scFv-DEC205, and the numbers of internalized bacteria after co-incubating with BMDCs and plasmid delivery to BMDCs in vitro [16]. The surface located scFvCD11c was observed to increase the cellular attachment and invasion to BMDCs in our study, while these increased effects were alleviated whenever the specific CD11c antibodies were present, indicating the specific interaction between $\mathrm{ScFv}-\mathrm{CD} 11 \mathrm{c}$ on L. plantarum and CD11c on BMDCs.

The increased cellular uptake of recombinant L. plantarum strains resulted in increased production of $\mathrm{CD} 11 \mathrm{c}^{+} \mathrm{GFP}^{+}$ BMDC cells, indicating that the newly constructed vector could be an option for future DNA vaccine delivery. Since the immune response was stimulated from the beginning 

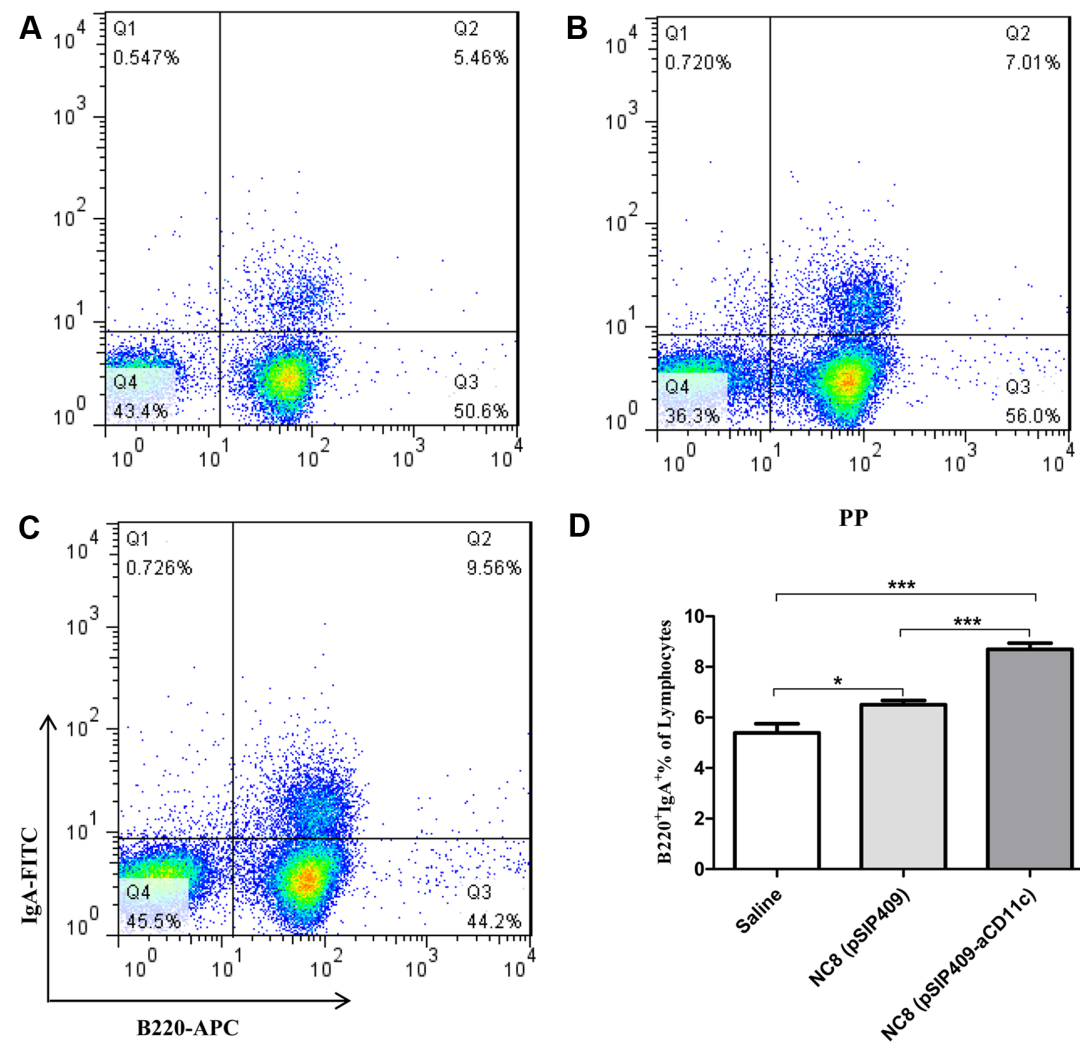

D

PP

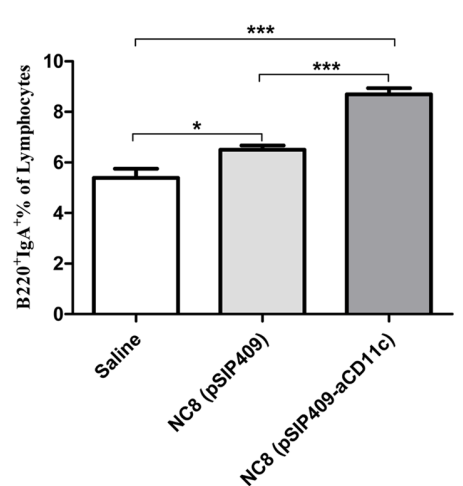

Fig. 7. Vaccination with DC-targeting L. plantarum resulted in the production of $\mathrm{B}_{2} 2 \mathrm{O}^{+} \mathrm{Ig} \mathrm{A}^{+} \mathrm{B}$ cells in the PP.

The flow scatter diagrams of (A) Saline, (B) NC8 (pSIP409) and (C) NC8 (pSIP409-aCD11c) are displayed. (D) The proportions of B220 IgA ${ }^{+}$B cells in the PPs were analyzed. The mean values \pm S.E.M. of three independent experiments are shown $\left({ }^{*} p<0.05\right.$ and $\left.{ }^{* * *} p<0.001\right)$.

of DC activation, followed by $\mathrm{T}$ cell differentiation and $\mathrm{B}$ cell activation, we therefore evaluated the effects of DCtargeting L. plantarum strains on DCs, T cells and B cells.

Consistent with a previous study in which the DCtargeting L. plantarum strain induced the activation of DC cells [6], our results also showed that the NC8 (pSIP409aCD11c) strains could efficiently stimulate the maturation of DCs. Since the differentiation of Th cells was usually represented as the production of cellular cytokines, such as IFN- $\gamma$, IL-4 and IL-17A, which were typical secreted cytokines representing Th1, Th2 and Th17 subtypes, respectively, we also determined the percentages of $\mathrm{T}$ cells producing these cytokines by FACS and the increased production of IL-4- and IL-17-producing T cells indicated the activation of Th2 and Th17 cells. Followed by the differentiation of Th cells, another important step for humoral immune response was the activation of B cells to produce IgA antibody, therefore the percentages of $\mathrm{IgA}^{+} \mathrm{B} 220^{+} \mathrm{B}$ cells were also measured and the results showed that the DC-targeting recombinant also increased the percentages of $\operatorname{IgA}^{+} \mathrm{B} 220^{+} \mathrm{B}$ cells, indicating its immunological modulation on the host immune system.

\section{Acknowledgments}

This work was supported by the The National Key Research and Development Program of China (2017YFD0501000, 2017YFD0500400), the National Natural Science Foundation of China (31602092, 31672528), Science and Technology Research Project of Jilin Provincial Education Department (JJKH20170316KJ), Science and Technology Development Program of Jilin Province (20160519011JH, 20170204034NY, 20180201040NY) and Special Funds for Industrial Innovation of Jilin Province (2016C063).

\section{Conflict of Interest}

The authors have no financial conflicts of interest to declare. 


\section{References}

1. Lechardeur D, Sohn KJ, Haardt M, Joshi PB, Monck M, Graham RW, et al. 1999. Metabolic instability of plasmid DNA in the cytosol: a potential barrier to gene transfer. Gene Ther. 6: 482-497.

2. Vijaya Kumar SG, Singh SK, Goyal P, Dilbaghi N, Mishra DN. 2005. Beneficial effects of probiotics and prebiotics on human health. Die Pharmazie 60: 163-171.

3. Liu L, Zhang W, Song Y, Wang W, Zhang Y, Wang T, et al. 2018. Recombinant Lactococcus lactis co-expressing $\mathrm{OmpH}$ of an M cell-targeting ligand and IBDV-VP2 protein provide immunological protection in chickens. Vaccine 36: 729-735.

4. Mancha-Agresti P, de Castro CP, Dos Santos JSC, Araujo MA, Pereira VB, LeBlanc JG, et al. 2017. Recombinant Invasive Lactococcus lactis Carrying a DNA Vaccine Coding the Ag85A Antigen Increases INF-gamma, IL-6, and TNF-alpha Cytokines after Intranasal Immunization. Front. Microbiol. 8: 1263.

5. Almeida JF, Breyner NM, Mahi M, Ahmed B, Benbouziane B, Boas PC, et al. 2016. Expression of fibronectin binding protein A (FnBPA) from Staphylococcus aureus at the cell surface of Lactococcus lactis improves its immunomodulatory properties when used as protein delivery vector. Vaccine 34: 1312-1318.

6. Sun Y, Qian J, Xu X, Tang Y, Xu W, Yang W, et al. 2018. Dendritic cell-targeted recombinant Lactobacilli induce DC activation and elicit specific immune responses against G57 genotype of avian H9N2 influenza virus infection. Vet. Microbiol. 223: 9-20.

7. Yang WT, Li QY, Ata EB, Jiang YL, Huang HB, Shi CW, et al. 2018. Immune response characterization of mice immunized with Lactobacillus plantarum expressing spike antigen of transmissible gastroenteritis virus. Appl. Microbiol. Biotechnol. 19: $8307-8318$.

8. Rigaux P, Daniel C, Hisbergues M, Muraille E, Hols P, Pot B, et al. 2009. Immunomodulatory properties of Lactobacillus plantarum and its use as a recombinant vaccine against mite allergy. Allergy 64: 406-414.

9. van Baarlen $P$, Troost FJ, van Hemert $S$, van der Meer $C$, de Vos WM, de Groot PJ, et al. 2009. Differential NF-kappaB pathways induction by Lactobacillus plantarum in the duodenum of healthy humans correlating with immune tolerance. Proc. Nat. Aca. Sci. USA 106: 2371-2376.

10. Figdor CG, van Kooyk Y, Adema GJ. 2002. C-type lectin receptors on dendritic cells and Langerhans cells. Nat. Rev. Immunol. 2: 77-84.

11. Moll H. 2003. Dendritic cells and host resistance to infection. Cell. Microbiol. 5: 493-500.

12. Bajtay Z, Csomor E, Sandor N, Erdei A. 2006. Expression and role of Fc- and complement-receptors on human dendritic cells. Immunol. lett. 104: 46-52.

13. Ejaz A, Ammann CG, Werner R, Huber G, Oberhauser V,
Horl S, et al. 2012. Targeting viral antigens to CD11c on dendritic cells induces retrovirus-specific $\mathrm{T}$ cell responses. PLoS One 7: e45102.

14. Kanazawa N. 2007. Dendritic cell immunoreceptors: C-type lectin receptors for pattern-recognition and signaling on antigen-presenting cells. J. Dermatol. Sci. 45: 77-86.

15. Zanoni I, Granucci F. 2010. Regulation of antigen uptake, migration, and lifespan of dendritic cell by Toll-like receptors. J. Mol. Med. 88: 873-880.

16. Christophe M, Kuczkowska K, Langella P, Eijsink VG, Mathiesen G, Chatel JM. 2015. Surface display of an antiDEC-205 single chain Fv fragment in Lactobacillus plantarum increases internalization and plasmid transfer to dendritic cells in vitro and in vivo. Microb. Cell Fact. 14: 95.

17. Sorvig E, Mathiesen G, Naterstad K, Eijsink VG, Axelsson L. 2005. High-level, inducible gene expression in Lactobacillus sakei and Lactobacillus plantarum using versatile expression vectors. Microbiology 151: 2439-2449.

18. Guimaraes V, Innocentin S, Chatel JM, Lefevre F, Langella P, Azevedo V, et al. 2009. A new plasmid vector for DNA delivery using lactococci. Genet. Vaccines Ther. 7: 4.

19. Han X, Wang L, Li W, Li B, Yang Y, Yan H, et al. 2015. Use of green fluorescent protein to monitor Lactobacillus plantarum in the gastrointestinal tract of goats. Braz. J. Microbiol. 46: 849-854.

20. Que YA, Haefliger JA, Francioli P, Moreillon P. 2000. Expression of Staphylococcus aureus clumping factor A in Lactococcus lactis subsp. cremoris using a new shuttle vector. Infect. Immun. 68: 3516-3522.

21. Almeida JF, Mariat D, Azevedo V, Miyoshi A, de Moreno de LeBlanc A, Del Carmen S, et al. 2014. Correlation between fibronectin binding protein A expression level at the surface of recombinant lactococcus lactis and plasmid transfer in vitro and in vivo. BMC Microbiol. 14: 248.

22. Perone MJ, Larregina AT, Shufesky WJ, Papworth GD, Sullivan ML, Zahorchak AF, et al. 2006. Transgenic galectin-1 induces maturation of dendritic cells that elicit contrasting responses in naive and activated T cells. J. Immunol. 176: 7207-7220.

23. Lee S, Miller SA, Wright DW, Rock MT, Crowe JE, Jr. 2007. Tissue-specific regulation of CD8+ T-lymphocyte immunodominance in respiratory syncytial virus infection. J. Virol. 81: 2349-2358.

24. Mancha-Agresti P, Drumond MM, Carmo FL, Santos MM, Santos JS, Venanzi F, et al. 2017. A new broad range plasmid for DNA delivery in eukaryotic cells using lactic acid bacteria: in vitro and in vivo assays. Mol. Ther. Methods Clin. Dev. 4: 83-91.

25. Yagnik B, Padh H, Desai P. 2016. Construction of a new shuttle vector for DNA delivery into mammalian cells using non-invasive Lactococcus lactis. Microbes Infect. 18: 237-244. 